\title{
The Size Distribution of Dust toward HD 210121 as Determined from Extinction
}

Kristen A. Larson

Western Washington University, kristen.larson@wwu.edu

M.J. Wolff

W. G. Roberge

D. C. B. Whittet

L. He

Follow this and additional works at: https://cedar.wwu.edu/physicsastronomy_facpubs

Part of the Astrophysics and Astronomy Commons

\section{Recommended Citation}

Larson, Kristen A.; Wolff, M. J.; Roberge, W. G.; Whittet, D. C. B.; and He, L., "The Size Distribution of Dust toward HD 210121 as Determined from Extinction" (2000). Physics \& Astronomy. 1.

https://cedar.wwu.edu/physicsastronomy_facpubs/1 


\title{
THE SIZE DISTRIBUTION OF DUST TOWARD HD 210121 AS DETERMINED FROM EXTINCTION
}

\author{
K. A. Larson, ${ }^{1,2}$ M. J. WolfF, ${ }^{3}$ W. G. Roberge, ${ }^{1}$ D. C. B. Whittet, ${ }^{1}$ and L. $\mathrm{HE}^{1}$ \\ Received 1999 August 13; accepted 1999 November 16
}

\begin{abstract}
Several observations suggest that the molecular cloud toward HD 210121 contains an enhanced relative abundance of small dust grains. In particular, the value of the ratio of total-to-selective extinction $\left(R_{V}\right)$ is unusually low. In this paper, we estimate the size distribution of dust grains in this line of sight from the extinction curve observed in the near-infrared through the ultraviolet. We use the maximum entropy method (MEM) to find the smoothest possible size distribution consistent within a $\chi^{2}$ confidence level fit to the extinction data. While MEM has been shown to be a powerful tool in modeling average extinction curves, we show in this paper that MEM can also be a useful technique in modeling individual lines of sight that differ significantly from Galactic averages. We review existing data for HD 210121 and calculate the dust-to-gas ratio in order to constrain the amount of material used in the grain model. We present size distributions for both two-component models of silicate and graphite and threecomponent models that include amorphous carbon. We compare HD 210121 with the average diffuse interstellar medium and with three other high-latitude lines of sight. The grain-size distribution toward HD 210121 contains a relative excess of grains with radius $a<0.1 \mu \mathrm{m}$ as well as a relative deficiency of grains with radius $a>0.1 \mu \mathrm{m}$ as compared with the average diffuse interstellar medium and other clouds at high latitude.
\end{abstract}

Subject headings: dust, extinction — ISM: individual (DBB 80) — stars: individual (HD 210121)

\section{INTRODUCTION}

HD 210121 is a normal B3 V star behind a remarkable cloud at high Galactic latitude. The foreground cloud toward HD 210121 was first identified by Désert, Bazell, \& Boulanger (1988) as a region with excess $100 \mu \mathrm{m}$ emission from dust associated with molecular gas. In the decade since this initial detection, the dust and gas content of this cloud (called DBB 80 or IREC 80 ) has been studied extensively. Existing data include ultraviolet (Welty \& Fowler 1992) and infrared (Larson, Whittet, \& Hough 1996) extinction curves, broadband polarimetry (Larson et al. 1996), optical spectral lines of atomic and molecular gas-phase species (Welty \& Fowler 1992; de Vries \& van Dishoeck 1988; Gredel et al. 1992), and CO maps (Gredel et al. 1992; van Dishoeck et al. 1991). The cloud is at $(l, b) \sim\left(57^{\circ},-45^{\circ}\right)$ and is located about $150 \mathrm{pc}$ below the Galactic plane, or twice the molecular scale height (Welty \& Fowler 1992). Chemical models (Stark \& van Dishoeck 1994) and observations of the low $I(100 \mu \mathrm{m}) / N\left(\mathrm{H}_{\text {tot }}\right)$ ratio (Welty \& Fowler 1992) suggest that the interstellar ultraviolet (UV) radiation field incident on the surface of the cloud is reduced by a factor of 2 compared with $I_{\mathrm{UV}}$ in the Galactic plane. More than $90 \%$ of the total hydrogen and the wide variety of molecular species in this line of sight are contained in a single foreground cloud, thus yielding a particularly simple geometry (Welty \& Fowler 1992). DBB 80 is a translucent cloud with about 1 mag of visual extinction but has high molecular abundances and a density more typical of dark clouds.

The most notable features of this line of sight are an extremely steep UV extinction curve and a very small value

\footnotetext{
${ }^{1}$ Department of Physics, Applied Physics, and Astronomy, Rensselaer Polytechnic Institute, Troy, NY 12180.

${ }_{2}^{2}$ Present address: Department of Physics and Astronomy, Western Washington University, Bellingham, WA 98225; larsonk@physics. wwu.edu.

${ }^{3}$ Space Science Institute, 1540 30th Street, Suite 23, Boulder, CO 80303-1012; wolff@colorado.edu.
}

of the ratio of total-to-selective extinction $\left(R_{V}\right)$. Both of these aspects of the extinction curve toward HD 210121 suggest a relative excess of small grains in the foreground cloud. For this reason, we have constructed a model of the dust grain size distribution toward HD 210121 using the maximum entropy method (MEM) to fit the size distribution to the observed extinction curve. This method has been used by Kim, Martin, \& Hendry (1994, hereafter KMH) to determine the grain-size distributions for two average normalized extinction curves with the $R_{V}$ parameterization of Cardelli, Clayton, \& Mathis (1989, hereafter CCM). KMH present size distributions for the average extinction curve in the diffuse interstellar medium (ISM) where $R_{V}=3.1$, as well as for the curve with a larger value of $R_{V}=5.3$ that represents dense cloud environments. Our motivation for applying the methods described in KMH to the HD 210121 line of sight is twofold. First, we want to show that this method can be an effective indicator of the grain-size distribution in individual lines of sight. KMH model the average $R_{V}$-parameterized extinction curves of CCM, but this family of curves is only an average and thus masks real variations present among individual extinction curves. Because the extinction curve toward HD 210121 is unusual but is well determined from the near-infrared through the ultraviolet, it is an excellent choice for this method. Second, because HD 210121 has a small value of $R_{V}$ consistent with several other indicators of small-grain excess, it is an appropriate contrast to the average and large $R_{V}$ curves modeled by $\mathrm{KMH}$. We hope to discover whether the environmental conditions that modify grains in large $R_{V}$ regions act in the opposite sense in regions with small $R_{V}$. Furthermore, DBB 80 is just one of many known translucent molecular clouds at high Galactic latitude, and evidence is mounting to suggest that many high-latitude clouds have values of $R_{V}$ that are significantly lower than $R_{V}$ in the average diffuse ISM. Models of the grain-size distribution for small values of $R_{V}$ may give insight into both the evolution of dust in 
translucent high-latitude environments and the processes that lead to an enhanced abundance of small grains.

In $\S 2$, we review the evidence for a small-grain excess in the foreground cloud toward HD 210121. We present observational parameters that constrain the fitting procedure, including the dust-to-gas ratio in the foreground cloud. The best-fit grain-size distribution for HD 210121 using MEM to model the extinction curve is presented in $\S 3$. We compare the HD 210121 line of sight with the diffuse ISM and dense cloud averages of $\mathrm{KMH}$, as well as to three additional individual high-latitude lines of sight. Results from these comparisons are discussed in $\S 4$.

\section{OBSERVATIONAL CONSTRAINTS FOR GRAIN-SIZE MODELS OF HD 210121}

The essential component for any grain-size modeling is a well-determined extinction curve, and it is the features of the extinction curve toward HD 210121 that motivate this project. The UV extinction curve toward HD 210121, shown in Welty \& Fowler (1992), is extremely steep. Compared with other stars fitted with the Fitzpatrick \& Massa (1990) formulation, the linear continuum coefficients are the highest known, and the polynomial coefficient for the nonlinear UV rise is among the highest known. Variations among UV extinction curves are expected to be caused by variations in the relative abundance of small grains (e.g., Mathis 1996). Therefore, the steep UV extinction toward HD 210121 implies a relative excess of small grains in the foreground cloud. These small grains may provide a larger surface area per volume for molecule formation (Cardelli 1988), causing the high molecular column densities observed toward this star. In contrast with the abundance of small grains, the abundance of large grains remains mainly constant in the ISM. Near-infrared extinction curves, in contrast with UV extinction curves, are uniform across a range of conditions (Martin \& Whittet 1990), and the near-infrared extinction toward HD 210121 is consistent with this uniform shape.

\subsection{Ratio of Total-to-Selective Extinction}

Further evidence for small-grain abundance is found in the slope of the blue visual extinction curve, which is measured by the parameter

$$
R_{V} \equiv \frac{A_{V}}{E_{B-V}}
$$

called the ratio of total-to-selective extinction. This is the factor that allows absolute visual extinction $\left(A_{V}\right)$ to be calculated from observed reddening $\left(E_{B-V}\right)$, but it is also commonly assumed to be a gross measure of average grain size. Variations in $R_{V}$ are not caused by variations in the amount of visual extinction but instead are caused by variations in the normalized slope of the extinction curve, $E_{B-V} / A_{V} . R_{V}$ is near 3 in the diffuse ISM but is somewhat higher in dense clouds. This is because regions with a relative lack of small grains have a flatter slope toward wavelengths shorter than $V$, so $R_{V}$ (the inverse slope) increases. The lack of small grains in dense cloud sight lines is typically interpreted as a result of grain aggregation processes (Mathis 1996). In contrast with dense clouds, $R_{V}=2.1 \pm 0.2$ toward HD 210121 (Larson et al. 1996), which is among the smallest values of this parameter yet known. This small value of $R_{V}$ also is consistent with the small value of the wavelength of maximum polarization, $\lambda_{\max }$. In general, the relative scarcity of larger grains and the tendency of small grains to be poorly aligned means that interstellar polarization curves peak near the visible (e.g., Whittet 1996). A shift of the aligned grain-size distribution will produce a shift in the wavelength of maximum polarization. Toward HD 210121, $\lambda_{\max }=0.38 \pm 0.03 \mu \mathrm{m}$ (Larson et al. 1996), which is one of the smallest values of this parameter ever observed but is consistent with the correlation between $R_{V}$ and $\lambda_{\max }$ in the ISM.

In general, grains whose maximum extinction efficiency is in the visual are larger than grains that dominate extinction in the ultraviolet. However, because the environmental conditions that modify grain size are rapid and efficient, entire grain-size distributions tend to shift coherently (Mathis 1997). That is, variation in the extinction-curve shape in the visual usually implies analogous variation out into the ultraviolet. Indeed, the value of $R_{V}$ has been shown to correlate with the amount of UV extinction. CCM determined an average normalized extinction curve that is a function of only $R_{V}$ over a wide range of environmental conditions. Nevertheless, while the small value of $R_{V}$ agrees qualitatively with the high UV extinction toward HD 210121, the CCM curve for $R_{V}=2.1$ does not adequately match the observations, as shown in Figure 1. The observed normalized extinction curve determined by Welty \& Fowler (1992) is well represented by the Fitzpatrick \& Massa (1990) parameterization. We have used $R_{V}=2.1$ to calculate extinction $A_{\lambda} / A_{V}$ (solid line, Fig. 1) from the parameterized reddening $E_{\lambda-V} / E_{B-V}$. At $\lambda^{-1}=8 \mu \mathrm{m}^{-1}$, the CCM curve (dashed line) reproduces only $63 \%$ of the observed extinction excess above what is observed in diffuse lines of sight (dotted line). In addition, the low- $R_{V} \mathrm{CCM}$ curve has a stronger $2175 \AA$ bump than the diffuse average, but the actual bump toward HD 210121 is rather weak. The inability of the CCM curve for $R_{V}=2.1$ to fit the observed extinction may mean that the environmental processes that modify grain sizes in this cloud are size selective in some way. On the other hand, it may simply mean that the conditions in the cloud toward HD 210121 are outside the range for which the CCM curve was determined.

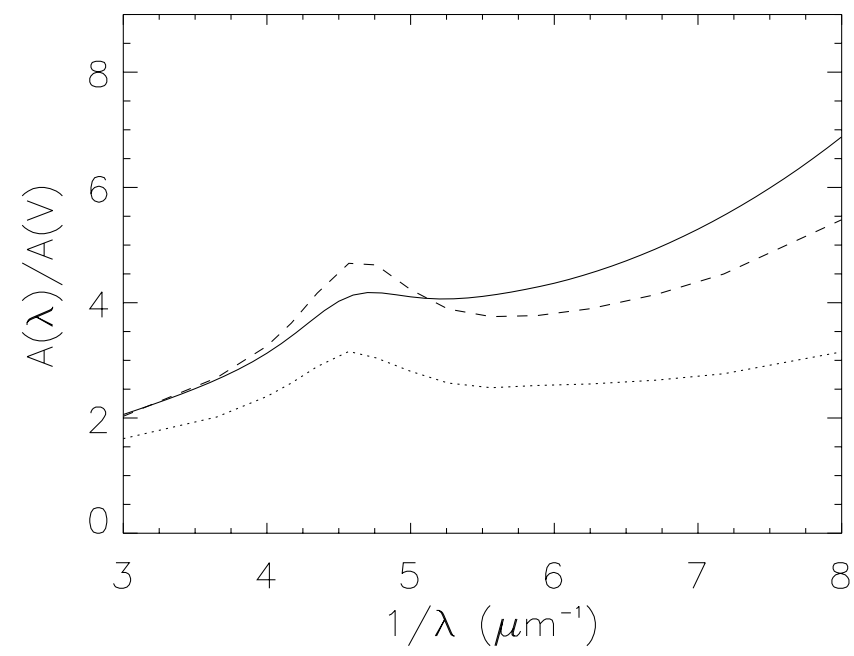

FIG. 1.-Normalized UV extinction curve toward HD 210121 calculated from parameterized observed color excess of Welty \& Fowler (1992) using $R_{V}=2.1$ (solid line). Observed extinction is compared with the CCM curve for $R_{V}=2.1$ (dashed line). The diffuse Galactic ISM average $\left(R_{V}=\right.$ $3.1)$ is shown as the dotted line. 


\subsection{Dust-to-Gas Ratio}

A great deal of evidence supports the hypothesis that the high-latitude cloud DBB 80 contains an unusually high relative excess of small grains. Armed with this evidence, we determine a model of the grain-size distribution that fits the observations in order to quantify this effect. However, for the grain-size distribution to be determined from the shape of the extinction curve, the dust-to-gas ratio must be known to constrain the amount of mass in the dust. One measure of the dust-to-gas ratio is $A_{V} / N\left(\mathrm{H}_{\text {tot }}\right)$, which depends also on the extinction efficiency per unit dust mass. The ratio of $A_{V} / N\left(\mathrm{H}_{\text {tot }}\right)$ is more relevant to the dust-to-gas ratio than $E_{B-V} / N\left(\mathrm{H}_{\text {tot }}\right)$ is, because the value of differential extinction, $E_{B-V}$, may be proportional to the amount of absolute extinction via an unusual value of the ratio $R_{V}(\mathrm{Kim} \&$ Martin 1996). For example, the low value of $E_{B-V} / N\left(\mathrm{H}_{\mathrm{tot}}\right)$ in the $\rho$ Oph line of sight is partially offset by the high ratio $R_{V}=4.2$ (Bohlin, Savage, \& Drake 1978).

Kim \& Martin (1996) show that, in models in which the total grain mass is conserved, the maximum acceptable value of $A_{V} / N\left(\mathrm{H}_{\mathrm{tot}}\right)$ increases slightly with increasing $R_{V}$. The mass-normalized distribution of the $R_{V}=5.3$ extinction curve has a value of $A_{V} / N\left(\mathrm{H}_{\mathrm{tot}}\right)$ that is $20 \%$ larger than the value at $R_{V}=3.1(\mathrm{KMH})$. In other words, models of high- $R_{V}$ extinction curves use less grain material than models of the average extinction curve where $R_{V}=3.1$. On the other hand, data compiled by Kim \& Martin (1996) show that lines of sight with large $R_{V}$ tend to have dust-togas ratios near the canonical average of $A_{V} / N\left(\mathrm{H}_{\mathrm{tot}}\right)=0.53$ $\times 10^{-21} \mathrm{mag} \mathrm{cm}^{2}$ (Bohlin et al. 1978), but, where $3.0<$ $R_{V}<4.4$, the dust-to-gas ratios span a much larger range and are on average greater than both the average at large $R_{V}$ and the canonical average. While this observational evidence for correlation between $R_{V}$ and $A_{V} / N\left(\mathrm{H}_{\mathrm{tot}}\right)$ is intriguing, it is not strong. There is a stronger correlation, however, between $R_{V}$ and the dust-to-molecular gas ratio if $\mathrm{CH}$ is used to trace the molecular component $\mathrm{H}_{2}$. The ratio $A_{V} / N(\mathrm{CH})$ is high at large $R_{V}$ and low at small $R_{V}$ (Cardelli 1988). The reason for this may be a combination of the dust-to-total gas ratio and the $\mathrm{CH} / \mathrm{H}_{2}$ ratio. The result of this correlation means that if $R_{V}$ is overestimated in a particular line of sight (such as assuming $R_{V}=3.1$ toward HD 210121), a fraction of the molecular gas component would be unaccounted for. In addition, the value of $A_{V}$ would be overestimated. Both of these effects lead to a severe overestimation of the dust-to-gas ratio, $A_{V} / N\left(\mathrm{H}_{\text {tot }}\right)$.

Li \& Greenberg (1998) have suggested that the value of $A_{V} / N\left(\mathrm{H}_{\mathrm{tot}}\right)$ toward HD 210121 may be high. One might expect a high dust-to-gas ratio at small $R_{V}$ in contrast with the lower than average $A_{V} / N\left(\mathrm{H}_{\text {tot }}\right)$ at $R_{V}>4.4$ in the Kim \& Martin (1996) compilation. However, the data for HD 210121 do not support this hypothesis. The column density of the $\mathrm{H}_{2}$ gas can be determined from observations of trace molecules. Since the conversion factor between $\mathrm{CO}$ and $\mathrm{H}_{2}$ has been shown to vary widely in observations at high latitude (Magnani \& Onello 1995) and in chemical models of translucent clouds (van Dishoeck 1994), it is a poor tracer of molecular gas in these environments. Instead, methods using $\mathrm{CH}$ have been shown to be more accurate tracers of molecular gas in the translucent environment (van Dishoeck \& Black 1989). Using $\mathrm{CH}$ and the dark cloud ratio, de Vries \& van Dishoeck (1988) determine $N\left(\mathrm{H}_{2}\right)=9.6$ $\times 10^{20} \mathrm{~cm}^{-2}$. Compared with $N(\mathrm{H} \mathrm{I})=2.9 \times 10^{20} \mathrm{~cm}^{-2}$
(Welty \& Fowler 1992; Heiles \& Habing 1974), most of the hydrogen in this line of sight is in molecular form. This high molecular fraction is consistent with the correlation between low $R_{V}$ and high $N(\mathrm{CH}) / A_{V}$. The total hydrogen gas column density in this line of sight is thus $N\left(\mathrm{H}_{\text {tot }}\right)=$ $N(\mathrm{H} \mathrm{I})+2 N\left(\mathrm{H}_{2}\right)=2.2 \times 10^{21} \mathrm{~cm}^{-2}$. Reddening in this line of sight is $E_{B-V}=0.38 \mathrm{mag}$ (Welty \& Fowler 1992); thus, the absolute extinction is $A_{V}=R_{V} E_{B-V}=0.80 \mathrm{mag}$. Combining the observations of the dust and gas content in this cloud, we determine that the dust-to-gas ratio in DBB 80 is

$$
\frac{A_{V}}{N\left(\mathrm{H}_{\mathrm{tot}}\right)}=0.36 \times 10^{-21} \mathrm{mag} \mathrm{cm}{ }^{2},
$$

about 30\% lower than the standard value from Bohlin et al. (1978). The largest source of uncertainty in this calculation is the molecular column density. The $\mathrm{CH} / \mathrm{H}_{2}$ ratio is predicted to be higher in regions with a low UV radiation field, which means that $N\left(\mathrm{H}_{2}\right)$ determined from average empirical ratios may be overestimated (de Vries \& van Dishoeck 1988). However, the molecular column density would have to be overestimated by more than $50 \%$ if the dust-to-gas ratio is close to the standard average. In addition, the rich molecular spectrum, low incident UV radiation field, and shielding by a steep UV extinction curve are all consistent with a high molecular fraction.

\section{SIZE MODELS DETERMINED FROM EXTINCTION}

Following Kim et al. (1994), we use MEM to infer the grain-size distribution from the extinction curve toward HD 210121. We model the grains as bare spheres with the optical constants of silicate and graphite (Mathis, Rumpl, \& Nordsieck 1977). MEM provides the smoothest grain-size distribution that is compatible with the extinction curve within a $\chi^{2}$ target confidence level. The distribution determined with MEM will match a given template function unless deviations from it are required by the data. A power law with exponential decay, $n(a) \propto a^{-3.5} \exp \left(-a / a_{b}\right)$ from 0.003 to $2.7 \mu \mathrm{m}(\mathrm{KMH})$, is used as the template. The MEM model is ideal for a problem whose solution (grain-size distribution) has more unknowns than data points (extinction wavelengths). We use this method and the implementation of KMH to model the line of sight toward HD 210121. An evolutionary model of the size distribution from the diffuse ISM into dense clouds as a reduction of the relative number of small particles with $a<0.1 \mu \mathrm{m}$ is verified in the KMH models. By modeling the HD 210121 line of sight, we investigate whether the evolutionary processes that result in fewer small grains at high $R_{V}$ have the opposite effect at low $R_{V}$. Because the CCM $R_{V}$-dependent extinction curve for $R_{V}=2.1$ does not adequately represent the observed UV curve toward this star, the results of KMH cannot necessarily be extrapolated to HD 210121.

The grain-size distribution toward HD 210121 is determined by fitting the calculated extinction from the size model to the observed near-infrared, optical, and parameterized UV extinction data from Larson et al. (1996; also Table 1) and Welty \& Fowler (1992; also Table 2). Normalized color excess is converted to extinction by

$$
\frac{A_{\lambda}}{A_{V}}=\frac{1}{R_{V}}\left(\frac{E_{\lambda-V}}{E_{B-V}}\right)+1,
$$


TABLE 1

NEAR-INFRARED AND OPTICAL EXTINCTION

\begin{tabular}{|c|c|c|c|c|c|c|c|}
\hline Star & $E_{V-J}$ & $E_{V-H}$ & $E_{V-K}$ & $E_{V-L}$ & $R_{V}$ & $A_{V}$ & Reference \\
\hline HD $210121 \ldots \ldots$ & 0.68 & 0.76 & 0.80 & 0.85 & 2.1 & 0.80 & 1 \\
\hline HD $145631 \ldots \ldots$ & 0.58 & 0.68 & 0.73 & 0.81 & 3.8 & 0.84 & 2 \\
\hline HD $154445 \ldots . .$. & 0.87 & 0.91 & 1.01 & 1.00 & 2.8 & 1.11 & 3 \\
\hline HD $203532 \ldots \ldots$ & 0.73 & 0.83 & 0.88 & 0.88 & 2.8 & 0.89 & 3 \\
\hline
\end{tabular}

REFERENCES.-(1) Larson et al. 1996; (2) K. A. Larson \& D. C. B. Whittet 2000, in preparation; (3) Whittet \& van Breda 1980.

using the ratio of total-to-selective extinction toward HD $210121\left(R_{V}=2.1\right.$, Larson et al. 1996). To constrain the amount of mass in the dust, we assume the dust-to-gas ratio calculated in $\S 2.2$. The best-fit size distributions derived for the silicate and graphite components toward HD 210121 are shown as solid and dashed lines, respectively, in the lower panel of Figure 2. The figures in this paper present grain size as the mass distribution $m(a) a$ normalized to the mass of hydrogen in the same volume. The template function is shown as the dot-dashed line in the lower panel of Figure 2. The upper panel of Figure 2 shows the extinction curves corresponding to the silicate and graphite components (solid and dashed lines, respectively). The total extinction (heavy line) is shown compared with the extinction observations (circles). For purposes of comparison, Figure 3 shows analogous results for the average diffuse ISM curve of CCM with $R_{V}=3.1$ and the standard dustto-gas ratio of Bohlin et al. (1978).

The two-component size model for HD 210121 uses $\mathrm{Si}$ / $\mathrm{H}=3.55 \times 10^{-5}$, or $11 \%$ more than our MEM solution for the diffuse ISM, and $\mathrm{C} / \mathrm{H}=2.16 \times 10^{-4}$, or $28 \%$ less than the diffuse ISM. While this may appear odd at first glance, a closer inspection shows that the disparity occurs because the steeper UV rise and weaker $2175 \AA$ bump of HD 210121 are fitted more robustly by more Si and less C. Constraining the two-component model to use only $90 \%$ or less of the Si forces the MEM algorithm to use more small graphite grains, which worsens the fit across the bump. Our two-component model uses all of the solar abundance of $\mathrm{Si}$ (Grevesse \& Anders 1989) but is within the solar abundance of C $\left(4.0 \times 10^{-4}\right.$; Grevesse et al. 1991) even if $35 \%$ of the carbon is in the gas phase (Cardelli et al. 1996). On the other hand, if the cosmic abundance of $\mathrm{C}$ is only about $70 \%$ of the solar abundance (Mathis 1996), then this model (along with many other models) will have difficulty reproducing the observed extinction. We also consider the slightly more realistic three-component model, in which the continuum opacity provided by the medium and large graphite grains is replaced by amorphous carbon (AMC). This "substitution" allows one to restrict the graphite component to the small sizes believed to be involved in producing the $2175 \AA$ bump. We use the BE1 optical constants of
Rouleau \& Martin (1991) for AMC. In Figures 4 and 5, we show plots analogous to Figures 2 and 3 for the results of three-component models with silicates (solid lines), AMC (dotted lines), and graphite (dashed lines).



FIG. 2.-HD 210121 two-component size distribution. The lower panel shows the mass distribution relative to the mass of hydrogen. Silicate and graphite components are shown as solid and dashed lines, respectively. The template function is shown as the dot-dashed line. The upper panel shows the corresponding extinction curves from each component. The sum (heavy line) is shown compared with the observed extinction (circles).

TABLE 2

UV EXTINCTION-CURVE Fit PARAMETERS

\begin{tabular}{|c|c|c|c|c|c|c|c|}
\hline Star & $c_{1}$ & $c_{2}$ & $c_{3}$ & $c_{4}$ & $\gamma$ & $\lambda_{0}^{-1}$ & Reference \\
\hline HD $210121 \ldots . .$. & -2.493 & 1.538 & 2.405 & 0.873 & 1.085 & 4.603 & 1 \\
\hline HD $145631 \ldots \ldots$ & 2.178 & 0.214 & 3.700 & 0.493 & 0.906 & 4.568 & 2 \\
\hline HD $154445 \ldots \ldots$ & 1.480 & 0.24 & 4.91 & 0.51 & 1.05 & 4.567 & 3 \\
\hline HD $203532 \ldots \ldots$ & 0.779 & 0.592 & 3.137 & 0.938 & 1.047 & 4.620 & 2 \\
\hline
\end{tabular}

REFERENCES.- (1) Welty \& Fowler 1992; (2) this paper; (3) Jenniskens \& Greenberg 1993. 


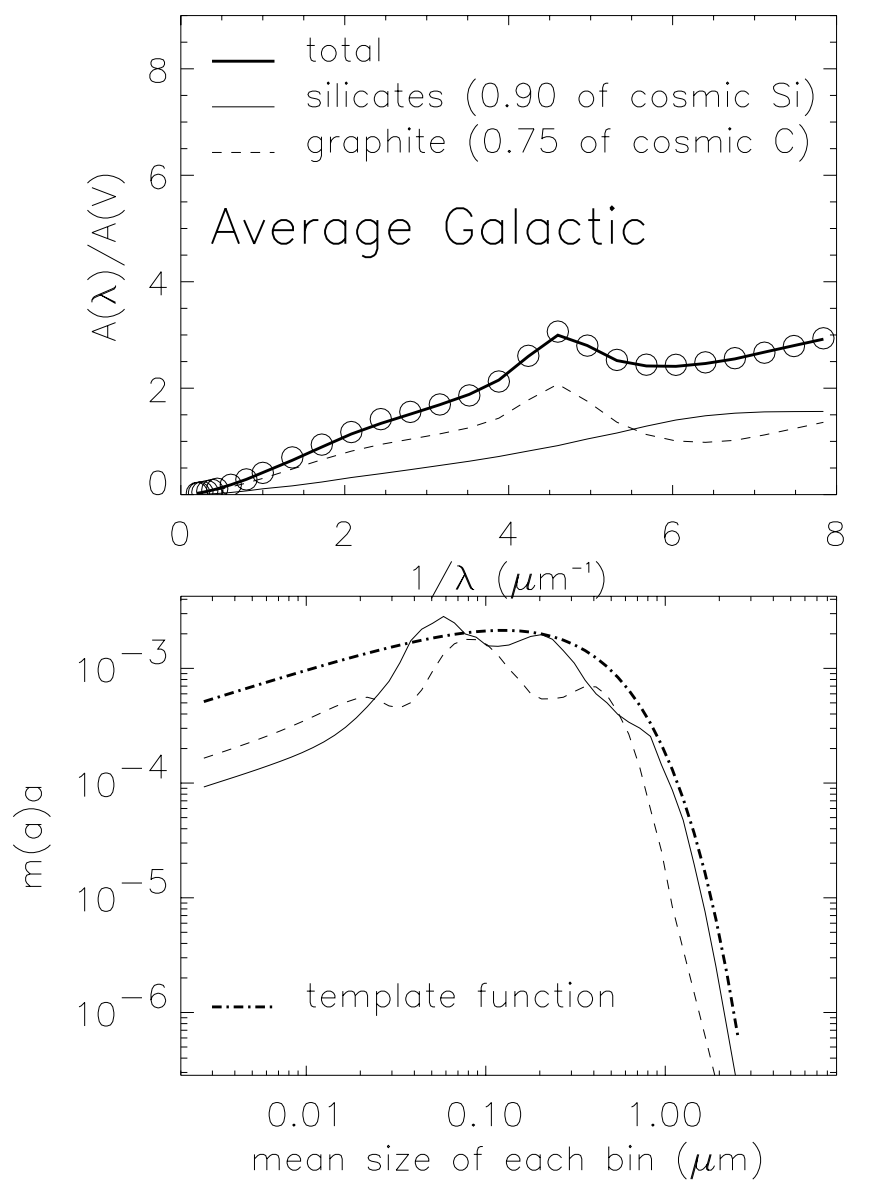

FIG. 3.-Same as Fig. 2, but for average diffuse ISM curve

In addition to HD 210121 and the average diffuse ISM curve, we also fit grain-size distributions to the extinction curves of three additional high-latitude stars. Our goal is to investigate whether the unusual structure of the size distribution toward HD 210121 is a common property of highlatitude dust. We choose the stars HD $145631[(l, b)=$ $(354.7,+22.5)]$, HD $203532(309.5,-31.5)$, and HD $154445(19.3,+22.9)$ for comparison with HD 210121. The optical data for these stars are listed in Table 3. The photometry and spectral type for HD 210121 are from Welty \& Fowler (1992), while the data for the three comparison stars are from the Mermilliod \& Mermilliod (1994) database. Blue visual reddening is calculated using the intrinsic colors from Schmidt-Kaler (1982). All of these stars were observed with the International Ultraviolet Explorer (IUE). The UV extinction curve for HD 154445 was determined by Aiello et al. (1988) and parameterized by Jenniskens \& Greenberg (1993). For the other two stars, we

TABLE 3

Optical Stellar Data

\begin{tabular}{cccccc}
\hline \hline Star & Spectral Type & $V$ & $B-V$ & $E_{B-V}$ & Reference \\
\hline HD $210121 \ldots \ldots$ & B3 V & 7.67 & 0.20 & 0.38 & 1 \\
HD $145631 \ldots \ldots$ & B9 V & 7.59 & 0.15 & 0.22 & 2 \\
HD $154445 \ldots \ldots$ & B1 V & 5.64 & 0.16 & 0.39 & 2 \\
HD 203532..... & B3 IV & 6.37 & 0.12 & 0.32 & 2 \\
\hline
\end{tabular}
1994.

REFERENCES.- (1) Welty \& Fowler 1992; (2) Mermilliod \& Mermilliod

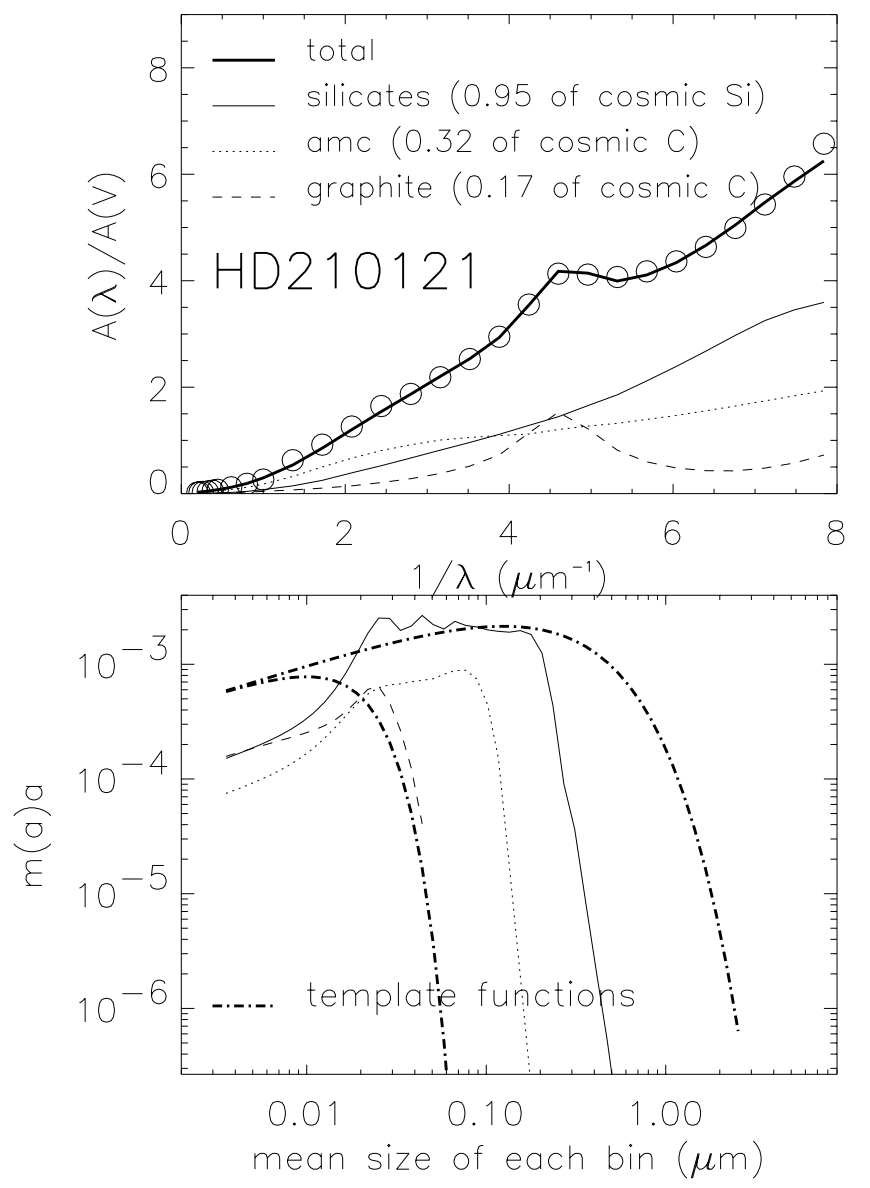

Fig. 4.-HD 210121 three-component size distribution. The lower panel shows the mass distribution relative to the mass of hydrogen. Silicate, AMC, and graphite components are shown as solid, dotted, and dashed lines, respectively. Template functions are shown as the dot-dashed lines. The upper panel shows the corresponding extinction curves from each component. The sum (heavy line) is shown compared with the observed extinction (circles).

calculate the UV extinction curves with the standard pair method: program stars are compared with stars that have low reddening and similar spectral type to calculate the amount of extinction relative to a reference band. The parameterization of the UV extinction toward these stars and HD 210121 is listed in Table 2 in the Fitzpatrick \& Massa (1990) formulation.

Near-infrared photometry of the three stars was obtained at the South African Astronomical Observatory (SAAO) and published by Whittet \& van Breda (1980) or K. A. Larson \& D. C. B. Whittet (2000, in preparation). Data are combined with intrinsic colors to calculate color excess. Because HD 154445 and HD 203532 were measured on the SAAO Glass system, intrinsic colors from Wegner (1994) are used for these stars. HD 145631 and HD 210121 were measured on the SAAO Carter system, and therefore we use the intrinsic colors of Glass (1997) for these stars. The nearinfrared color excess data for all four stars are listed in Table 1. The values of $A_{V}$ and $R_{V}$, also listed in Table 1, are determined by extrapolating the near-infrared color excess data to $\lambda \rightarrow \infty$; see K. A. Larson \& D. C. B. Whittet (2000, in preparation) for more details on the infrared data and analysis. In the absence of any indicators of anomalous dust-to-gas ratios, we assume the standard value of Bohlin et al. (1978) in our models. The three-component size dis- 


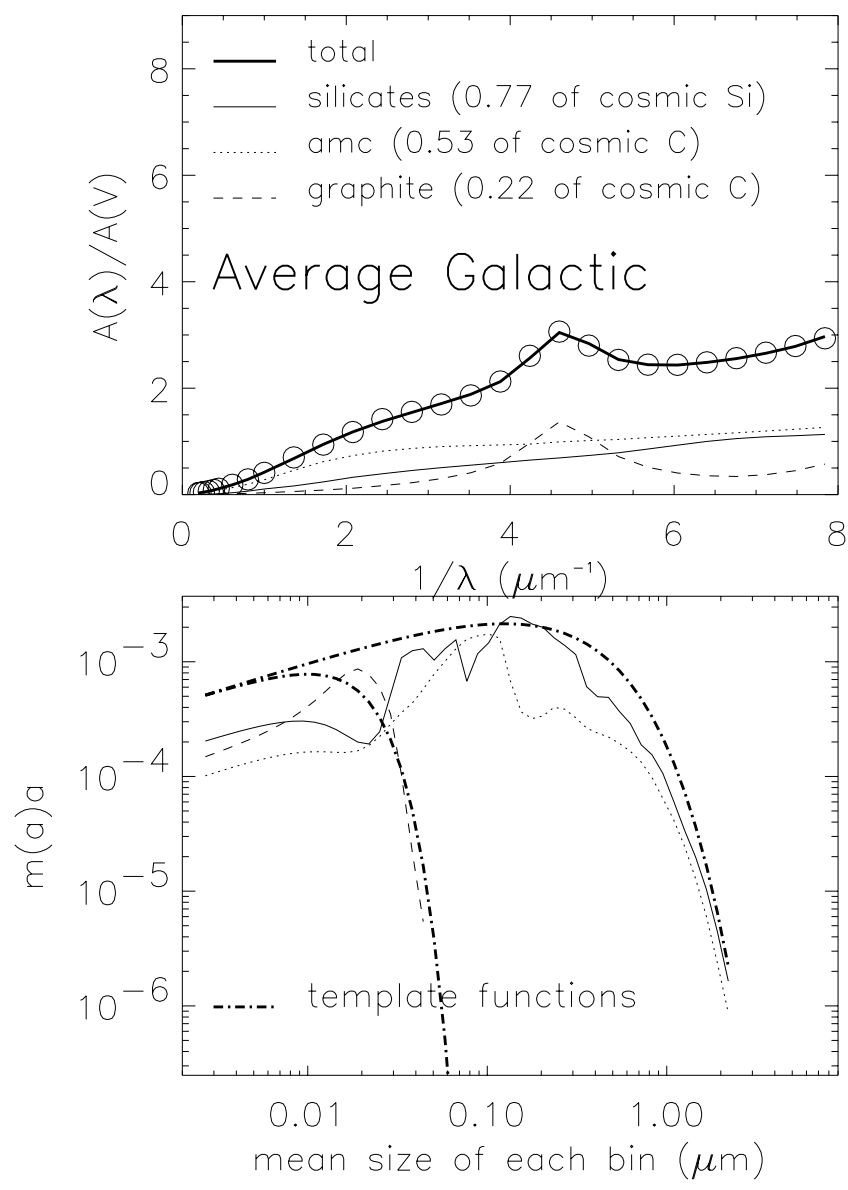

FIG. 5.-Same as Fig. 4, but for average diffuse ISM curve

tributions and the observed extinction toward the three comparison stars are shown in Figures 6, 7, and 8.

\section{DISCUSSION}

The shape of the size distribution toward HD 210121 is qualitatively similar to the diffuse ISM distribution. In the two-component models shown in Figures 2 and 3, the peaks in the size distribution of silicate grains near $a \simeq 0.05 \mu \mathrm{m}$ and $a \simeq 0.2 \mu \mathrm{m}$ are present in results for both HD 210121 and the average diffuse ISM models. The peaks in the graphite distribution near $a \simeq 0.08 \mu \mathrm{m}$ and $a \simeq 0.4 \mu \mathrm{m}$ are also present for both extinction curves. These features of the size distribution have been shown by KMH to be robust for this grain composition model in the diffuse ISM. While the shapes of the distributions are similar, the relative abundance of silicate grains smaller than $0.1 \mu \mathrm{m}$ is much higher toward HD 210121 than in the diffuse ISM. For example, the peak of the $a \simeq 0.05 \mu \mathrm{m}$ feature in the silicate distribution contains approximately twice as much mass as the same peak in the diffuse ISM curve and 10 times more mass than the same peak in the dense cloud curve with $R_{V}=5.3$ in $\mathrm{KMH}$. On the other hand, the relative abundance of small graphite grains toward HD 210121 and in the diffuse ISM is nearly equal. At the extreme small end of the grainsize distribution, the silicate structure at $a \simeq 0.025 \mu \mathrm{m}$ toward HD 210121 is absent in the diffuse ISM, while the graphite diffuse ISM structure at $a \simeq 0.02 \mu \mathrm{m}$ is missing toward HD 210121. However, it should be noted that the shape of the size distribution is not reliable for grain sizes

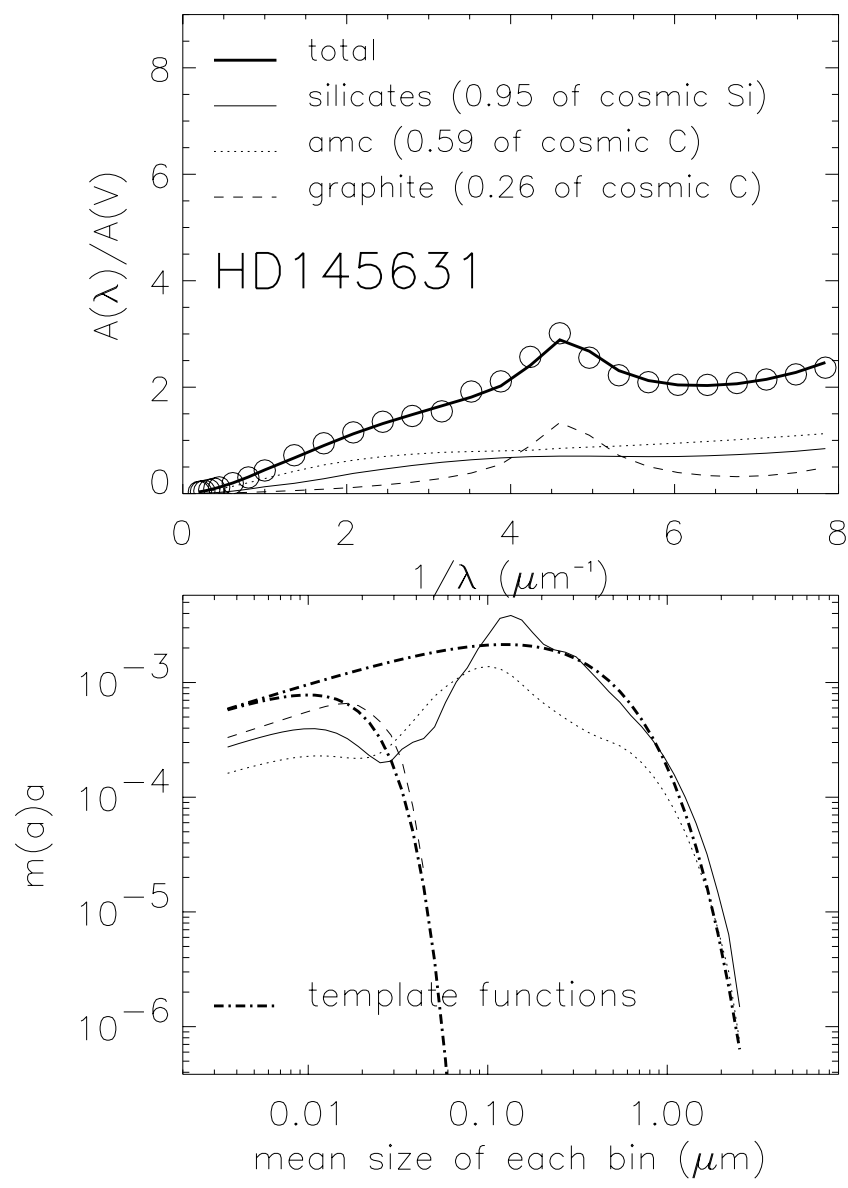

FIG. 6.-Same as Fig. 4, but for HD 145631

smaller than approximately $0.02 \mu \mathrm{m}$ for extinction data at $\lambda<0.1 \mu \mathrm{m}(\mathrm{KMH})$. Only the total mass is constrained for very small grains.

Most striking in a comparison of the size distributions in Figures 2 and 3 is the lack of large particles toward HD 210121. The qualitative structure at $a>0.1 \mu \mathrm{m}$ is present for both HD 210121 and the diffuse ISM, but the graphite particles at $a \sim 0.4 \mu \mathrm{m}$ are a factor of 10 less abundant and the silicates of the same size are 100 times less abundant toward HD 210121. In contrast, the high- $R_{V}$ model of $\mathrm{KMH}$ shows a reduction in the amount of small grains but no significant change in the shape of the large-grain distribution. Note that, while the functional form of the UV extinction toward HD 210121 is very unusual, the nearinfrared extinction toward this star has nearly the same shape as the Martin \& Whittet (1990) universal curve, as expected. Furthermore, KMH found that the shape of the size distribution of large grains is sensitive neither to small variations in the power-law index of the near-infrared extinction curve nor to the value of $R_{V}$. Therefore, the lack of large grains toward HD 210121 is somewhat surprising. Our model of the grain-size distribution toward HD 210121 confirms a relative excess of small grains in the foreground cloud and a shift toward smaller mean grain size, but we also find that the small mean grain size toward HD 210121 is due to both an excess of small grains and a lack of large grains in the cloud. These features of the size distribution are consistent with a lack of grain growth in this cloud, as suggested by Larson et al. (1996). In the three-component 


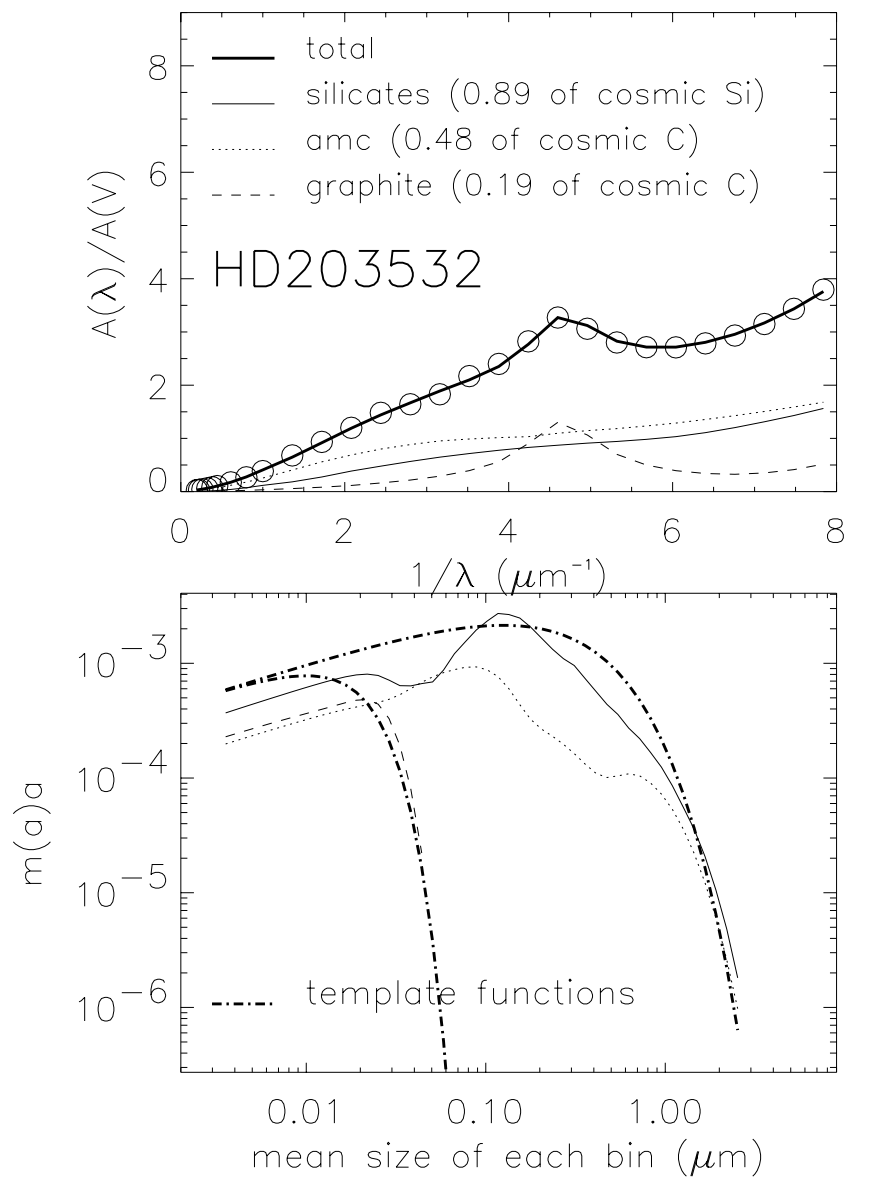

FIG. 7.- Same as Fig. 4, but for HD 203532

models of Figures 4 and 5, the excess of small grains $(a<0.1 \mu \mathrm{m})$ and the scarcity of large grains $(a>0.1 \mu \mathrm{m})$ are even more apparent. Both silicates and AMC show pronounced excess near $a \simeq 0.02 \mu \mathrm{m}$. It is interesting to note that the peak of the graphite component is shifted to slightly larger grain size for HD 210121. The lack of large grains is particularly striking in the three-component model. By repeating these models for other template functions, we find that the qualitative features of the size distribution toward HD 210121 are insensitive to the chosen template.

The grain-size distributions toward the three comparison high-latitude stars are not significantly different than in the diffuse ISM model. None of the three comparison distributions shows any significant excess of small grains or lack of large grains. This is not surprising given that the extinction curves of all three stars are unremarkable and are much more like the diffuse ISM curve than they are like the HD 210121 extinction curve. The value of $R_{V}$ toward HD 145631 is slightly high, and $R_{V}$ toward HD 154445 and HD 203532 are within the range expected in the diffuse ISM. All three of these comparison stars are located near large molecular cloud complexes associated with the Galactic plane: HD 145631 is near the $\rho$ Oph region, HD 154445 is near the Aquila Rift, and HD 203532 is near the highlatitude molecular clouds south of the Chamaeleon complex. HD 210121, on the other hand, is behind an isolated cloud that is tens of degrees from the dark clouds of the Galactic plane. None of the comparison sight lines exhibits the dramatic structures apparent in the size distribution of dust grains toward HD 210121.

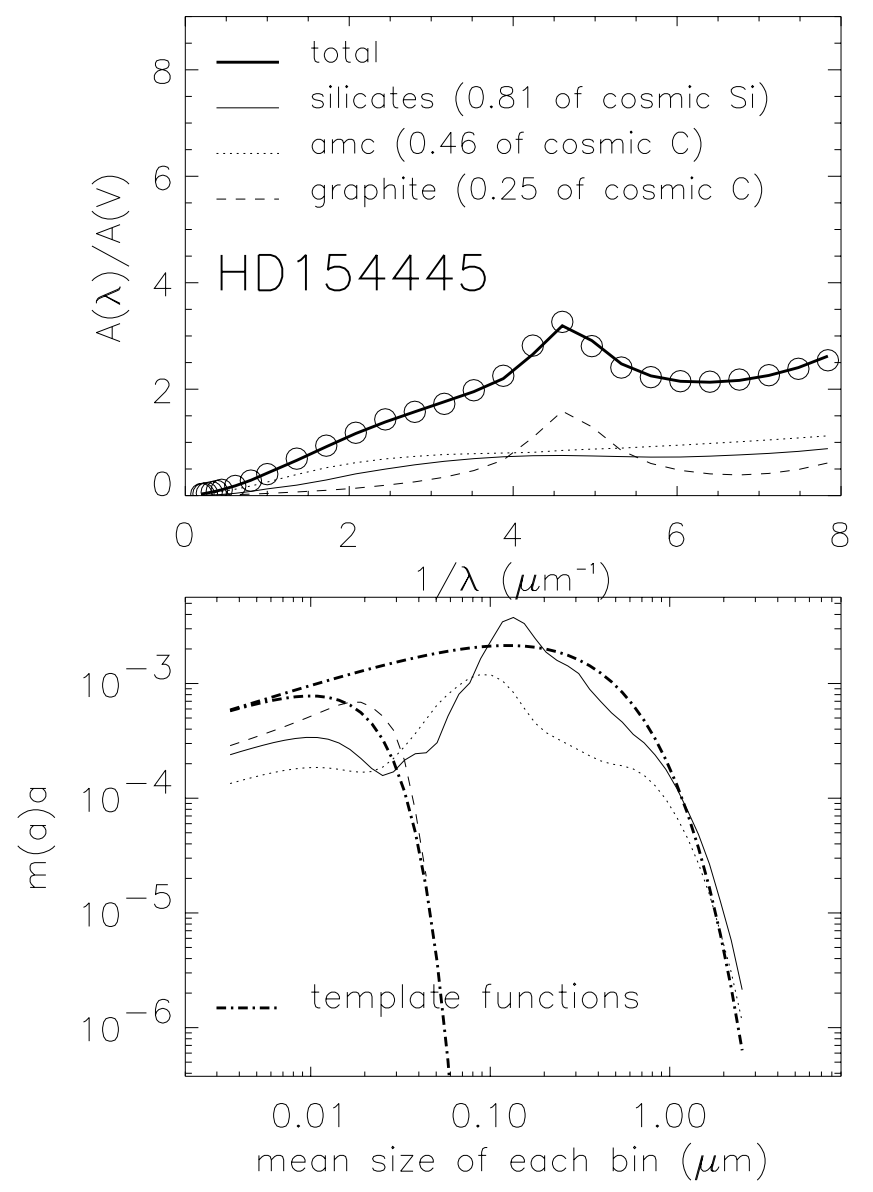

FIG. 8.-Same as Fig. 4, but for HD 154445

The cloud toward HD 210121 is just one of many translucent molecular clouds at high Galactic latitude. See Magnani, Hartmann, \& Speck (1996) for a compilation of known molecular clouds and review of cloud properties and Reach, Wall, \& Odegard (1998) for an atlas of high-latitude $100 \mu \mathrm{m}$ excess. While the average value of $R_{V}$ at high latitude is very similar to the value in the Galactic diffuse ISM, the values of $R_{V}$ at high latitude span a wide range and include a significant population of values that are much lower than the diffuse ISM average (K. A. Larson \& D. C. B. Whittet 2000, in preparation). Szomoru \& Guhathakurta (1999) find that the optical extinction in three large high-latitude regions of $100 \mu \mathrm{m}$ emission is more consistent with values of $R_{V}$ smaller than the diffuse ISM average. There is also some evidence that the variations in UV extinction correlate with distance from the Galactic plane. Kiszkurno-Koziej \& Lequeux (1987) suggest that the 2175 $\AA$ bump weakens and the slope of the far-UV curve steepens with increasing distance from the Galactic midplane. Because the distance to most high-latitude clouds is not well determined, it is difficult to categorize high-latitude clouds by their height above the Galactic plane. Among the four stars modeled in this paper, however, HD 210121 is at the highest latitude, and, at a height of $150 \mathrm{pc}$, the cloud toward HD 210121 is located farther away from the Galactic plane than most other high-latitude clouds. While the unusual grain-size distribution toward HD 210121 may be partially due to its distance from the Galactic plane (and distance from sources of UV radiation or disruptive processes), the extinction curve toward this star is unusual even among 
high-latitude stars. K. A. Larson \& D. C. B. Whittet (2000, in preparation) find no consistent correlation between the value of $R_{V}$ and Galactic latitude and conclude that local conditions must dominate variation in the size parameter $R_{V}$ at high latitude. Evidence for small $R_{V}$ at high latitude is still somewhat inconclusive, but if confirmed it will change our current understanding of the conditions and processes at work in these clouds.

\section{CONCLUSION}

In order to estimate the grain-size distribution toward HD 210121 from the observed extinction, we have used MEM as it was implemented by Kim et al. (1994). The motivating goal of this project is to use MEM on an individual observed extinction curve that does not match the average $R_{V}$-dependent curves of Cardelli et al. (1989). In addition, several indicators of a small-grain abundance toward HD 210121 allow us to extend the results of Kim et al. (1994) for average and large-grain environments to a line of sight with an excess of small grains. We find with both two-component and three-component models that the grain-size distribution toward HD 210121 shows an increase for grains $a<0.1 \mu \mathrm{m}$ and a marked decrease for grains $a>0.1 \mu \mathrm{m}$ as compared with the diffuse ISM average. With the low dust-to-gas ratio of HD 210121, our models use nearly all of the cosmic abundance of $\mathrm{Si}$ and about one-half of the cosmic abundance of $\mathrm{C}$. We compare the size distribution for HD 210121 with three other highlatitude lines of sight and find that the size distributions of grains toward the other high-latitude objects are very similar to the diffuse ISM average. In many ways, the foreground cloud to HD 210121 is unique. However, if recent indications of enhanced small-grain abundance away from the Galactic plane are confirmed, this line of sight may give insight into the evolution of these clouds and their role in the ISM.

We would like to thank Peter Martin for providing access to and advice about the MEM algorithm and its implementation. We also thank Paul Hendry for the source code to the MEM object library. K. A. L. acknowledges support from the US Department of Education Graduate Assistance Program and thanks Western Washington University for support during the preparation of this manuscript. M. J. W. acknowledges the support of NASA grant NAG 5-3904. D. C. B. W. acknowledges the support of NASA grant NAG 5-3376.
Aiello, S., Barsella, B., Chlewicki, G., Greenberg, J. M., Patriarchi, P., \& Perinotto, M. 1988, A\&AS, 73, 195

Bohlin, R. C., Savage, B. D., \& Drake, J. F. 1978, ApJ, 224, 132

Cardelli, J. A. 1988, ApJ, 335, 177

Cardelli, J. A., Clayton, G. C., \& Mathis, J. S. 1989, ApJ, 345, 245 (CCM)

Cardelli, J. A., Meyer, D. M., Jura, M., \& Savage, B. D. 1996, ApJ, 467, 334

Désert, F. X., Bazell, D., \& Boulanger, F. 1988, ApJ, 334, 815 (DBB)

de Vries, C. P., \& van Dishoeck, E. F. 1988, A\&A, 203, L23

Fitzpatrick, E. L., \& Massa, D. 1990, ApJS, 72, 163

Glass, I. S. 1997, Mon. Notes Astron. Soc. South Africa, 56, 100

Gredel, R., van Dishoeck, E. F., de Vries, C. P., \& Black, J. H. 1992, A\&A, 257,245

Grevesse, N., \& Anders, E. 1989, in AIP Conf. Proc. 183, Cosmic Abundances of Matter, ed. R. G. Lerner (New York: AIP), 1

Grevesse, N., Lambert, D. L., Sauval, A. J., van Dishoeck, E. F., Farmer, C. B., \& Norton, R. H. 1991, A\&A, 242, 488

Heiles, C., \& Habing, H. 1974, A\&AS, 14, 1

Jenniskens, P., \& Greenberg, J. M. 1993, A\&A, 275, 549

Kim, S.-H., \& Martin, P. G. 1996, ApJ, 462, 296

Kim, S.-H., Martin, P. G., \& Hendry, P. D. 1994, ApJ, 422, 164 (KMH)

Kiszkurno-Koziej, E., \& Lequeux, J. 1987, A\&A, 185, 291

Larson, K. A., Whittet, D. C. B., \& Hough, J. H. 1996, ApJ, 472, 755

Li, A., \& Greenberg, J. M. 1998, A\&A, 339, 591

Magnani, L., Hartmann, D., \& Speck, B. G. 1996, ApJS, 106, 447

Magnani, L., \& Onello, J. S. 1995, ApJ, 443, 169

Martin, P. G., \& Whittet, D. C. B. 1990, ApJ, 357, 113

\section{REFERENCES}

Mathis, J. S. 1996, in ASP Conf. Ser. 97, Polarimetry of the Interstellar Medium, ed. W. G. Roberge \& D. C. B. Whittet (San Francisco: ASP), 3 . 1997, in ASP Conf. Ser. 122, Stardust to Planetesimals, ed. Y. J. Pendleton \& A. G. G. M. Tielens (San Francisco: ASP), 87

Mathis, J. S., Rumpl, W., \& Nordsieck, K. H. 1977, ApJ, 217, 425

Mermilliod, J.-C., \& Mermilliod, M. 1994, Catalog of Mean UBV Data on Stars (New York: Springer)

Reach, W. T., Wall, W. F., \& Odegard, N. 1998, ApJ, 507, 507

Rouleau, F., \& Martin, P. G. 1991, ApJ, 377, 526

Schmidt-Kaler, T. 1982, in Landolt-Börnstein, Numerical Data and Functional Relationships in Science and Technology, ed. K. Schaifers \& H. H. Voigt (New York: Springer), 1

Stark, R., \& van Dishoeck, E. F. 1994, A\&A, 286, L43

Szomoru, A. \& Guhathakurta, P. 1999, AJ, 117, 2226

van Dishoeck, E. F. 1994, in ASP Conf. Ser. 58, The First Symposium on the Infrared Colors and Diffuse Interstellar Clouds, ed. R. M. Cutri \& W. B. Latter (San Francisco: ASP), 319

van Dishoeck, E. F., \& Black, J. H. 1989, ApJ, 340, 273

van Dishoeck, E. F., Black, J. H., Phillips, T. G., \& Gredel, R. 1991, ApJ, 366,141

Wegner, W. 1994, MNRAS, 270, 229

Welty, D. E., \& Fowler, J. R. 1992, ApJ, 393, 193

Whittet, D. C. B. 1996, in ASP Conf. Ser. 97, Polarimetry of the Interstellar Medium, ed. W. G. Roberge \& D. C. B. Whittet (San Francisco: ASP), 125

Whittet, D. C. B., \& van Breda, I. G. 1980, MNRAS, 192, 467 